and Behaviour Rating Scale (BRS) scores (estimate $=-0.39 ; \mathrm{p}=$ 0.012).

Conclusions Implementation of a neonatal pain and sedation protocol results in an increase in opiate prescription without affecting short-term outcome and neurodevelopmental performance of VLBWI at 12 months corrected age.

\section{P0-0455 RESISTIVE INDEX (RI) OF CEREBRAL ARTERIES IN VERY PRETERM INFANTS: REFERENCE VALUES AND IMPACT OF PATENT DUCTUS ARTERIOSUS}

${ }^{1} \mathrm{GM}$ Ecury-Goossen, 'MMA Raets, ${ }^{2} \mathrm{FA}$ Camfferman, ${ }^{3} \mathrm{~J}$ van Rosmalen, ${ }^{1} \mathrm{IKM}$ Reiss, ${ }^{4} \mathrm{P}$ Govaert, 'J Dudink. 'Department of Pediatrics, Division of Neonatology, Erasmus MC-Sophia Children's Hospital, Rotterdam, Netherlands; ${ }^{2}$ Department of Pediatrics Division of Neonatology, UZ Brussel, Rotterdam, Netherlands; ${ }^{3}$ Department of Biostatistics, Erasmus MC, Rotterdam, Netherlands; ${ }^{4}$ Department of Pediatrics, Koningin Paola Children's Hospital, Antwerp, Belgium

\subsection{6/archdischild-2014-307384.1096}

Background and aims We describe resistive index (RI) values in various cerebral arteries in a cohort of preterm infants born at $<29$ weeks gestation. We also aim to compare RI in various intracranial arteries, and to assess the relationship between RI and hemodynamically significant patent ductus arteriosus (PDA). Methods Preterm infants admitted to our NICU between May 2010 and January 2013 were enrolled. RI of internal carotid arteries, basilar artery, anterior cerebral artery, pial and striatal arteries was obtained in the first three days of life and weekly after that until discharge or death.

Results 771 examinations were performed in 235 infants. RI differed depending on which artery was insonated. RI was negatively correlated with measured time averaged velocity, except in the basilar artery. There was a significant difference in RI when comparing left (mean 0.86) to right sided (0.84) internal carotid artery $(\mathrm{p}=0.023)$. RI was lower in patients without PDA compared to patients with hemodynamically significant PDA, though this difference was small and not statistically significant in all arteries. There was no difference in pre- and postligation RI in patients who underwent PDA ligation. RI was not related to gestational age, postnatal age, SNAPPE II score or gender.

Conclusions For accurate follow-up and comparison of RI, it is important to examine the same artery. No definitive cut-off value for RI indicative for the presence of a hemodynamically significant PDA was found. Cerebral artery RI may have limited value as a clinical tool in the very preterm infant.

\section{PO-0456 MEASURING CEREBRAL AUTOREGULATION. A COMPARISON OF FREQUENCY - AND TIME DOMAIN ANALYSIS}

VR Eriksen, GH Hahn, G Greisen. Department of Neonatology, Rigshospitalet Copenhagen University Hospital, Copenhagen, Denmark

\subsection{6/archdischild-2014-307384.1097}

Background and aims Cerebral autoregulation (CA) is described by the relation between mean arterial blood pressure and cerebral blood flow. The aim of this study was to compare frequency - and time domain analysis - two conventional methods used to describe CA.

Methods We measured cerebral oxygenation (as a surrogate for cerebral blood flow) and mean arterial blood pressure in 60 preterm infants in the first day of life. Coherence and gain were calculated by means of frequency domain analysis. The cerebral oxyimetry index (COx) and the regression coefficient were calculated in the time domain. For both calculations $5 \mathrm{~min}$ windows were applied in 4-17 consecutive $10 \mathrm{~min}$ epochs of good quality signal in each infant. Analysis of variance was used to compare the intra-infant and inter-infant variation.

Results Discrimination among the infants was less good for coherence than for $\mathrm{COx}(\mathrm{F}=3.4$ vs. 8.8). The correlation between coherence and COx was poor (Pearson's $r=0.215$, $\mathrm{p}=0.097)$. Applying conventional thresholds for 'impaired' autoregulation resulted in different classifications (Chi2 $=3.78$, $\mathrm{p}=0.052$ ). In one extreme case, gain was $0.68 \mathrm{microM} / \mathrm{mmHg}$ - the highest of all the infants - while the regression coeffficient of the time-domain analysis was $-0.33 \mathrm{microM} / \mathrm{mmHg}$ - the lowest of all. This was due to cerebral oxygenation and arterial blood pressure being in counterphase.

Conclusions Time domain analysis gave better discrimination among infants, suggesting better precision. Also, a high gain and high coherence may arise spuriously when cerebral oxygenation decreases as blood pressure increases.

\section{PO-0457 ASSOCIATION BETWEEN EARLY VISUAL MOTION PERCEPTION IN VERY PRETERM CHILDREN AND NEURODEVELOPMENT AT 2.5 YEARS}

${ }^{1} \mathrm{Y}$ Fredriksson Kaul, ${ }^{2} \mathrm{~K}$ Rosander, ${ }^{2} \mathrm{C}$ von Hofsten, ${ }^{3} \mathrm{~B}$ Böhm, ${ }^{1} \mathrm{~K}$ Strand Brodd, 'L Hellström Westas. 'Department of Women's and Children's Health, Uppsala University, Uppsala, Sweden; ${ }^{2}$ Department of Psychology, Uppsala University, Uppsala, Sweden; ${ }^{3}$ Department of Women's and Children's Health, Karolinska Institute, Stockholm, Sweden

\subsection{6/archdischild-2014-307384.1098}

Introduction Young infants need visual motion perception to understand their environment. Gaze reflects the ability to track moving objects, and includes smooth pursuit (SP) eye movements, saccades and head movements.

Aim To investigate if early visual tracking ability in children born very preterm (VPT) is associated with later neurodevelopment.

Method The VPT infants $(n=68)$ had mean (SD) gestational age of 28.3 (2.6) weeks and birth weight 1154 (365) g. Ability to track moving objects was recorded at 4 months corrected age (CA) using a system combining electro-oculography and cameras recording head movements. Gaze and proportion of SP were measured. Tracking ability corresponding to at least the 10th percentile of a term born control group was defined as normal, and served as cut off for VPT infants. Neurodevelopment was assessed in VPT infants at 2.5 years (Bayley Scales of Infant Development III, BSID III).

Results At 4 months $37 \%$ and 25\% of VPT infants had subnormal SP and Gaze, respectively. The table shows group differences in BSID III subscale scores for Gaze and SP, between children with normal compared to subnormal early visual tracking ability.

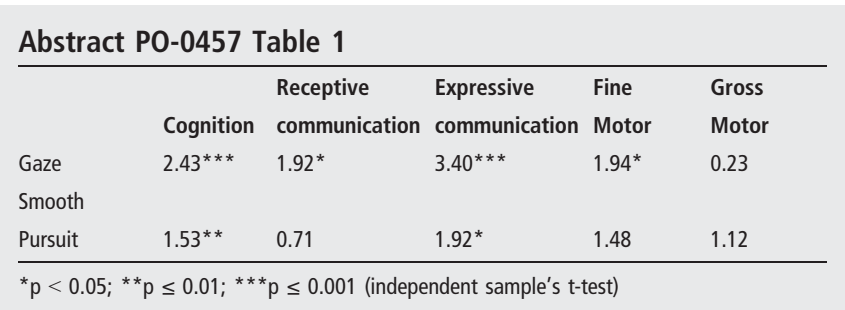

